\section{Military Technical College Kobry El-Kobbah, Cairo, Egypt}

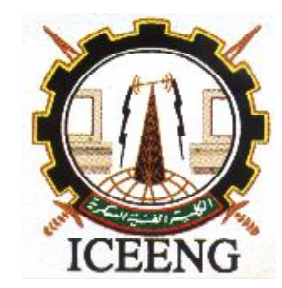

\title{
Design and Implementation of Impulse Radio Ultra-Wideband Transmitter
}

\author{
By \\ Osama Hassan Hussein Aly *, Ihab Adly **, Khaled A. Shehata *, Hani F. Ragai ***
}

\section{$\underline{\text { Abstract: }}$}

This paper illustrates the simulation, implementation and testing of an UWB transmitter based on Step Recovery Diode (SRD). For testing purposes, a square wave generator has been also implemented based on Colpitts oscillator and a clipping circuit that was used to trigger the monocycle pulse generator. The simulated output is a monocycle pulse waveform with 300 ps pulse width and acceptable symmetry in both amplitude and shape. Integrating the UWB transmitter and the Colpitts oscillator, the achieved output from this integration after implementation is a monocycle pulse waveform with 420 ps pulse width and acceptable symmetry in both amplitude and shape.

\section{Keywords:}

Ultra-Wideband Systems; pulse generator, SRD, monocycle pulse.

Arab Academy for Science, Technology and Maritime Transport, Cairo, Egypt CEO, Tegrom, Egypt

Electronics and Communications Department, Faculty of Engineering, Ain Shams University 


\section{Introduction:}

Ultra-Wideband (UWB) systems convey information by transmitting sub-nanosecond short pulses. These extremely short pulses produce ultra-wide bandwidth; thus, these systems are called ultra-wideband systems. According to the regulation of the Federal Communication Commission (FCC), a signal is defined as an UWB signal if its absolute bandwidth (BW) $\geq 500 \mathrm{MHz}$ or the fractional bandwidth $\left(\mathrm{B}_{f}\right) \geq 0.2$. The definition of $\mathrm{B}_{f}$ is [1]:

$$
B_{f}=\frac{f_{H}-f_{L}}{\left(f_{H}+f_{L}\right) / 2}
$$

UWB technology enables major enhancements in three wireless application areas: communications, radar systems and positioning / ranging. UWB technology can be utilized also over wire lines and cables such as CATV application. Each of these applications illustrates the unique value of UWB [2][3].

Gaussian monocycle and doublet pulses can be used in UWB systems. These kinds of pulses share together the common feature of very wideband spectrums. Gaussian pulse has a shape of Gaussian distribution. It is worth noting that Gaussian pulses remain Gaussian distributed when they pass through any linear systems. A general formula of Gaussian pulse is shown in equation (2), in which $\tau$ is a time constant [4].

$g_{0}(t)=-e^{-\left(\frac{t-t_{0}}{\tau}\right)}$

Nevertheless, spectrum of the monocycle pulse does not contain low - frequency components including DC. This feature can be utilized for some applications, as it enhances the design of other system components, among which are amplifiers, sampling down converters and antennas. Thus, better signal characteristics (i.e., more precisely defined pulse shape and reduced distortion for the transmitting and receiving signals) can be a lot easier to access.

Monocycle pulse has been generated by obtaining the first derivative of a Gaussian pulse under the following equation 0 .

$g_{1}(t)=-\frac{t-t_{1}}{\tau} e^{-\left(\frac{t-t_{0}}{\tau}\right)^{2}}$ 


\section{$\underline{\text { 2. Gaussian monocycle } \text { pg }_{\underline{S}}:}$}

A shaping filter can be used to transform the Gaussian pulse into a Gaussian monocycle pulse. Monocycle pulses have been generated by using SRD and combining two Gaussian pulses having 180-degree out of phase and certain time delay between them 0 . However, the pulse duration of the produced monocycle pulse is about twice as much as that of each individual Gaussian pulse.

Another method for generating Monocycle pulse introduced in [6], which uses a simple $\mathrm{RC}$ high-pass filter as a differentiator to generate the monocycle pulse directly.

The same technique to generate Monocycle pulse is used in [7], which uses an extra short circuit microstrip section to invert and delay the pulse to form a Gaussian monocycle pulse. The sectioned microstrip line is used to generate a reconfigurable monocycle pulse, as in [8].

To weigh among those techniques to adopt the most efficient one, this paper uses SRD and a short-circuited stub for generating the Gaussian pulse, which undergoes differentiation through RC high-pass filter to obtain a monocycle pulse. Fig. 1 shows the process of generating monocycle pulse generator.

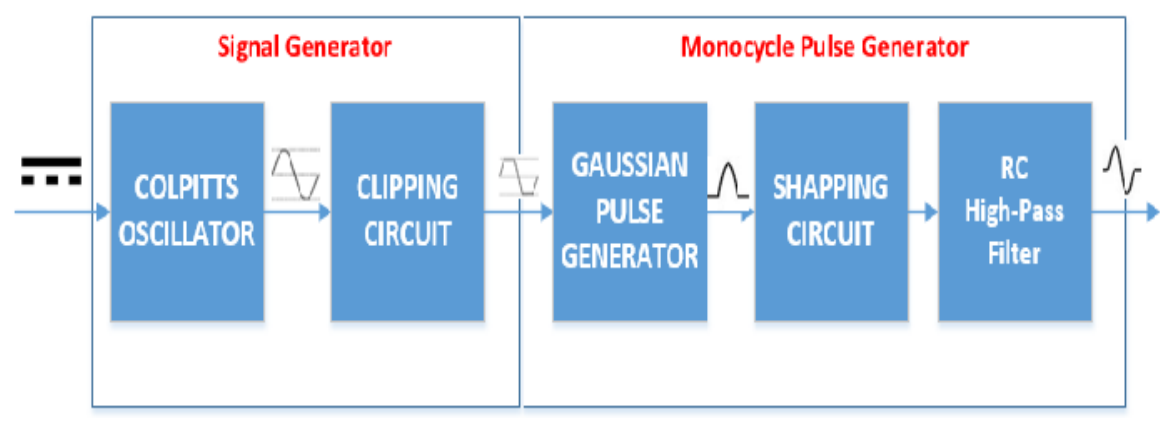

Figure (1): Block diagram of the monocycle pulse generator

\section{Circuit Description and Design:}

Fig. 2 shows the circuit diagram of a monocycle pulse generator. It comprises three parts: Gaussian pulse generator, shaping circuit and RC high-pass filter. The first part produces a Gaussian pulse in two stages. 


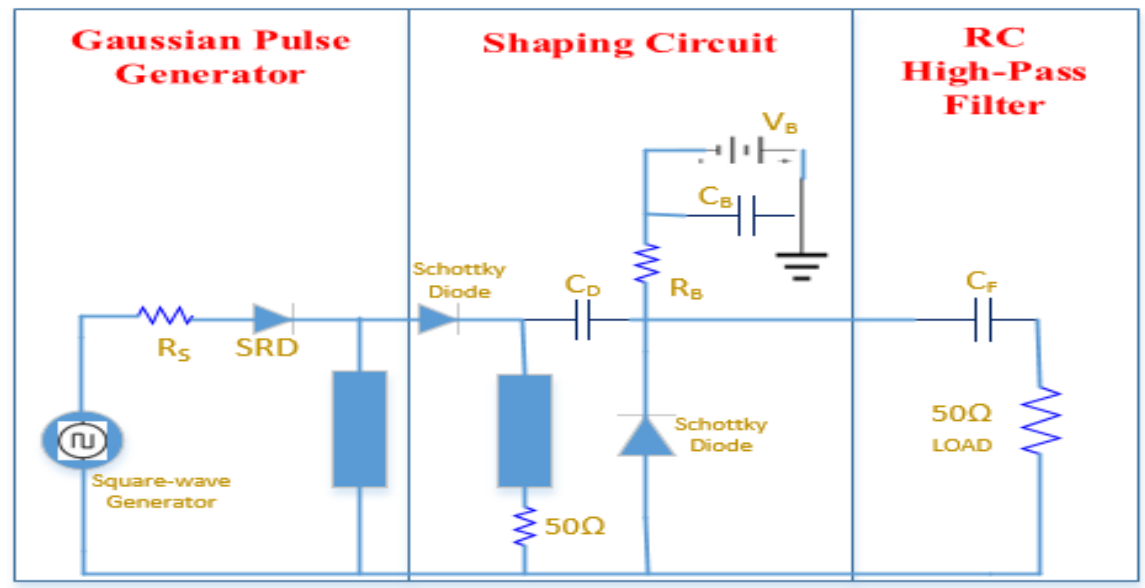

Figure (2): Designed monocycle pulse generator

First, a short transition time step function produced by SRD. Second, a Gaussian pulse shaped by merging the step function reflected from the short-circuited stub and the one at the junction between the SRD and the short-circuited stub. The produced Gaussian pulse width is determined by the length of the short-circuited stub as shown in Fig. 3, which is simulated by using Agilent-ADS (2014).

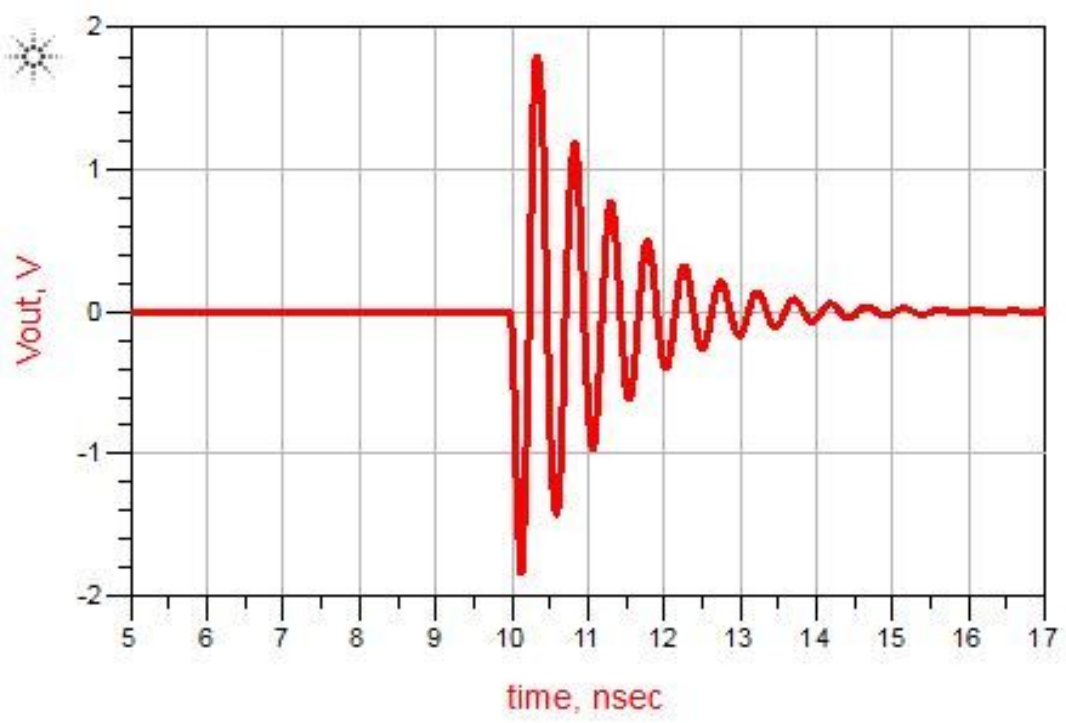

Figure (3): O/P of the Gaussian pulse generator

As shown in Fig. 3, a very large ringing appears when the shaping circuit is not used because there is no matching impedance between the Gaussian pulse generator and the RC network. Fig. 4 shows considerable removal of the ringing by using a short-circuited stub terminated with 50 resistor. 


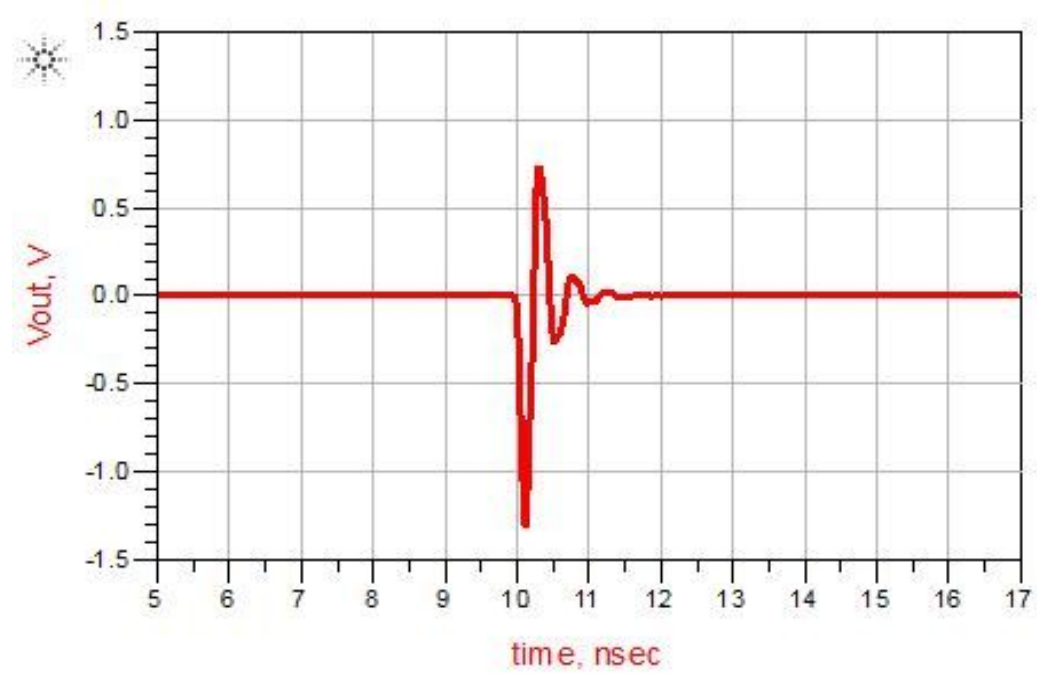

Figure (4): O/P after applying short-circuited stub terminated with 50 resistor

The ringing level is further minimized by using a series Schottky diode acting as a halfwave rectifier, allowing only the positive parts to pass through as shown in Fig. 5.

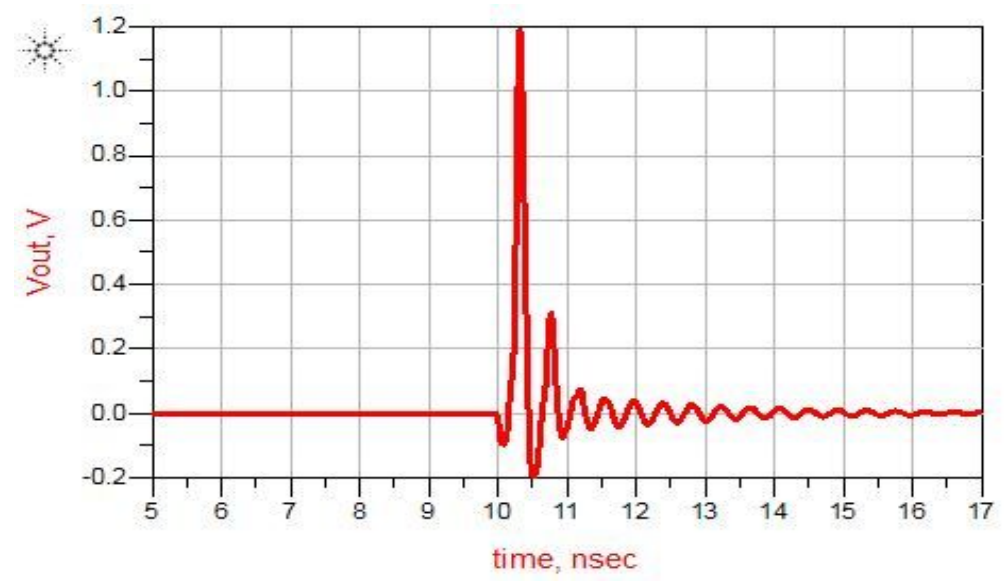

Figure (5): O/P after applying a series Schottky diode

Fig. 6 shows the final Gaussian waveform, with very small ringing, after passing through a shunt Schottky diode that acts as a fast switch allowing only the parts of the signal with amplitudes greater than a threshold level controlled by the DC bias voltage.

The RC high-pass filter is made up of a capacitor and a load resistor. The value of the capacitance is calculated by the load resistance and the desired time constant. This time constant can be approximately calculated from the duration of the Gaussian pulse and 
the settling time of the exponentially attenuated response of a step function to the RC network.

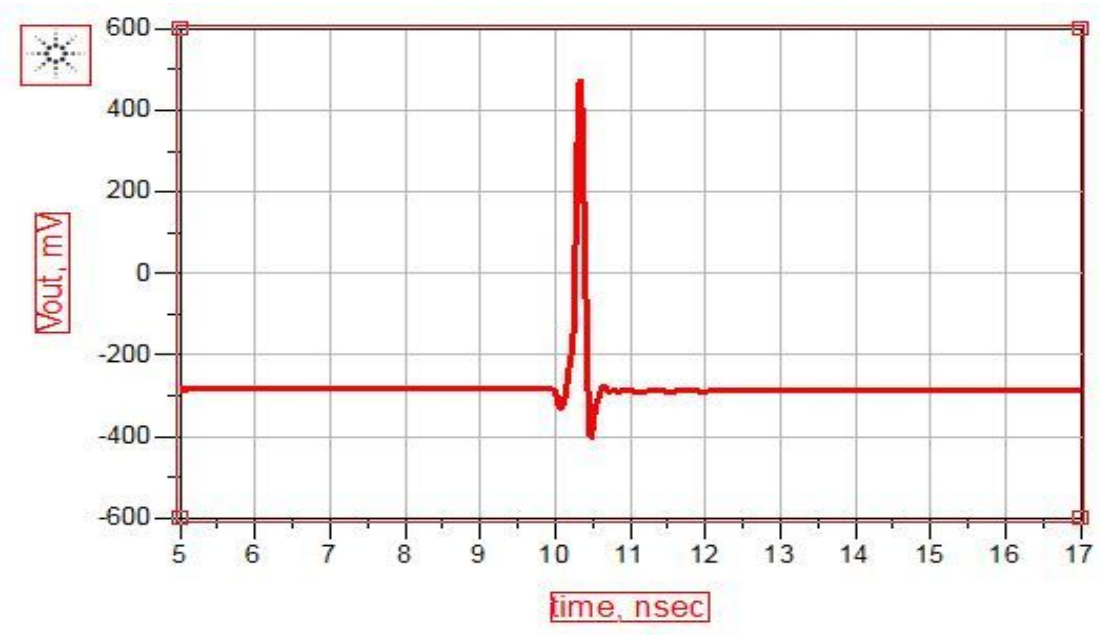

Figure (6): Final Gaussian pulse waveform

Fig. 7 shows the simulated monocycle pulse with enhanced reduced ringing and improved symmetry. The pulse width is $300 \mathrm{ps}$. The output monocycle pulse complies with the FCC regulations in which the PSD of the output frequency is $-44.9 \mathrm{dBm} / \mathrm{MHz}$ as shown in Fig. 8.

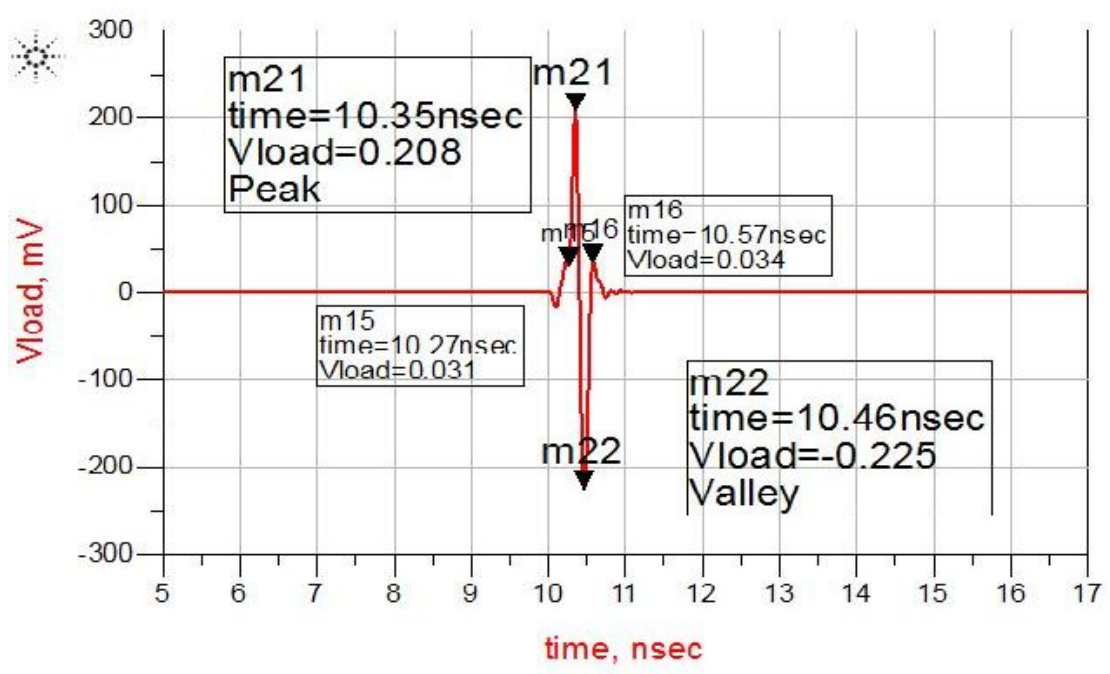

Figure (7): Simulated monocycle pulse 


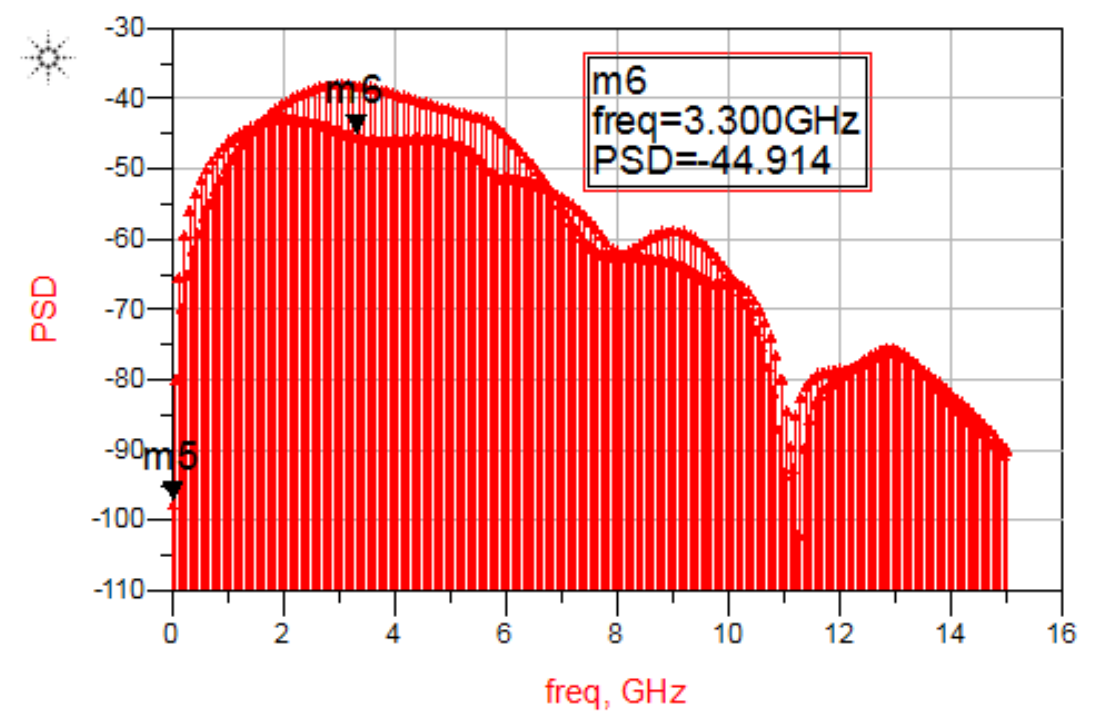

Figure (8): PSD of the monocycle pulse

\section{FABRICATION AND MEASUREMENT:}

The monocycle pulse generator was manufactured by using microstrip line on Rogers 6010 microwave laminates with relative dielectric constant of 10.7 and a thickness of $1.9 \mathrm{~mm}$. The used models of the SRD and Schottky diodes are SMMD840-SOT23-0S, MSS50, and 048-P86 respectively. They are manufactured by Cobham Metelics. Fig. 9 demonstrates the fabricated circuit.

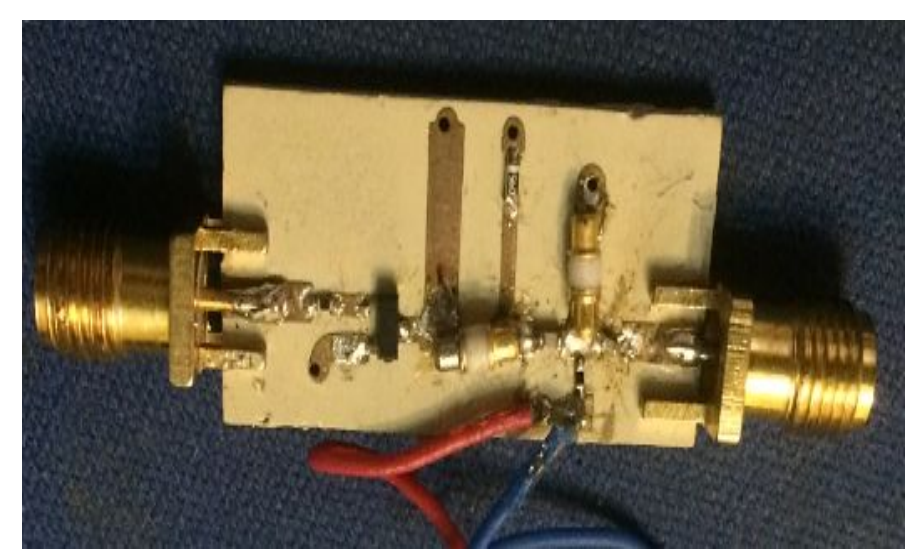

Figure (9): The fabricated circuit 


\section{INTEGRATED SIGNAL GENERATOR:}

In the process of integration, Colpitts oscillator model and a clipping circuit have been used to generate the desired square wave in order to trigger the monocycle pulse generator circuit with modified parameters to achieve matching throughout the new schematic. Fig. 10 shows the designed Colpitts oscillator circuit that uses a capacitive voltage divider network as its feedback source. The two capacitors, $\mathrm{C} 1$ and $\mathrm{C} 2$ are placed across a single common inductor, L. These components form the tuned tank circuit [9].

The advantage of this capacitive-circuit configuration type is that the frequency stability of the oscillator is improved along with a more simplified design with less self and mutual inductance within the tank circuit.

The Colpitts oscillator uses a single stage bipolar transistor amplifier as the gain element producing a sinusoidal output. The frequency of oscillations for a Colpitts oscillator is determined by the resonant frequency of the LC tank circuit and is given by the following equation.

$$
\mathrm{f}_{\mathrm{r}}=\frac{1}{\left(2 \pi \sqrt{\left(\mathrm{LC}_{\mathrm{T}}\right)}\right.}
$$

Where $\mathrm{C}_{\mathrm{T}}$ is the capacitance of $\mathrm{C}_{1}$ and $\mathrm{C}_{2}$ connected in series and is calculated by:

$$
\mathrm{C}_{\mathrm{T}}=\frac{\mathrm{C}_{1} * \mathrm{C}_{2}}{\mathrm{C}_{1}+\mathrm{C}_{2}}
$$

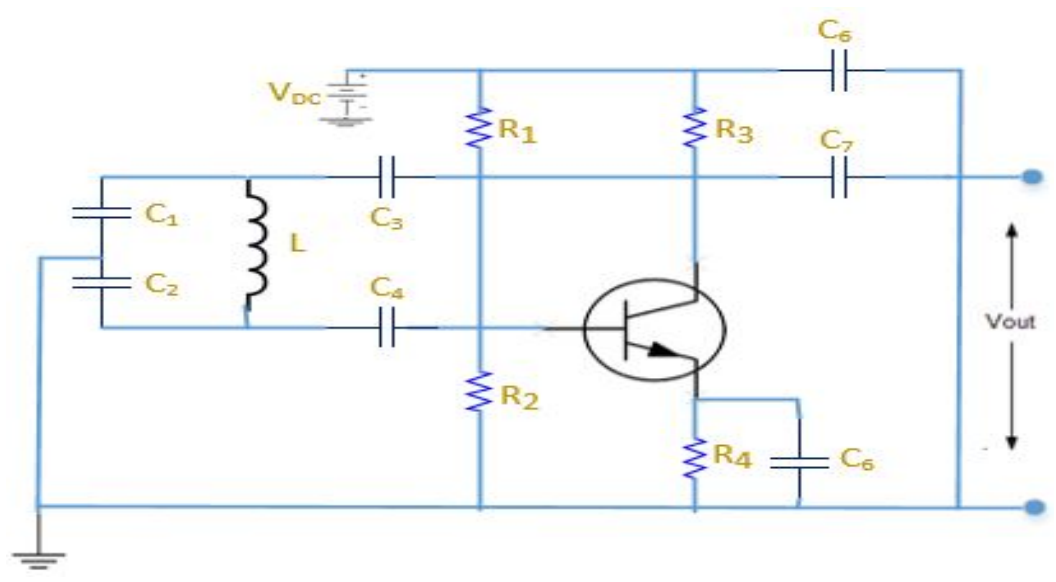

Figure (10): Designed Colpitts oscillator circuit

The output of the designed Colpitts oscillator is shown in Fig 11. 


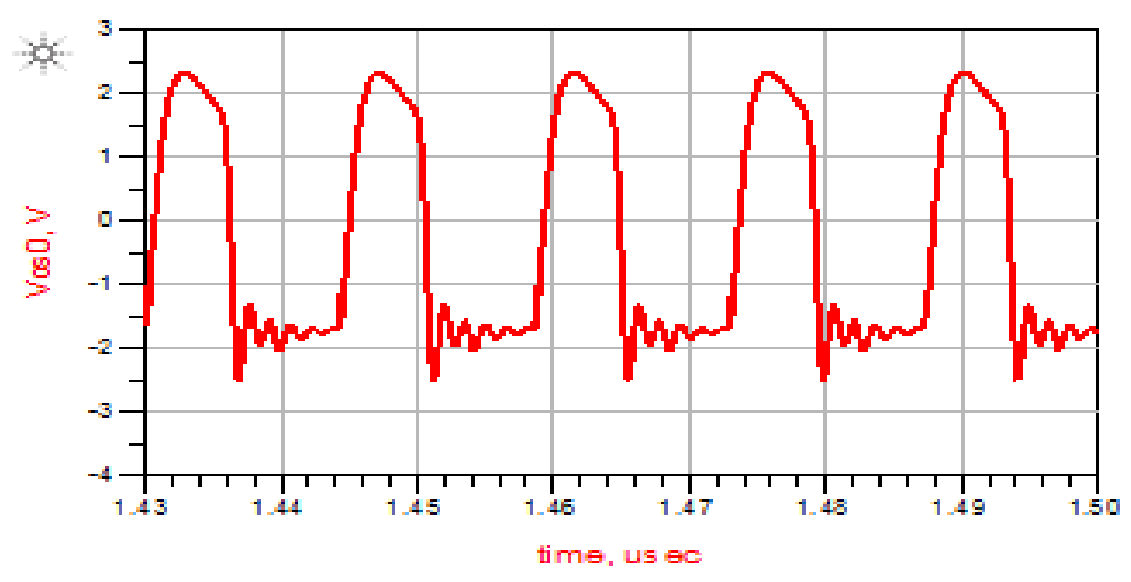

Figure (11): O/P of the designed Colpitts oscillator

A clipping circuit shown in Fig. 12 has been used to transform the output of the Colpitts oscillator into approximately squared wave to trigger the monocycle pulse generator circuit. Fig. 13 shows the output of the designed clipping circuit.

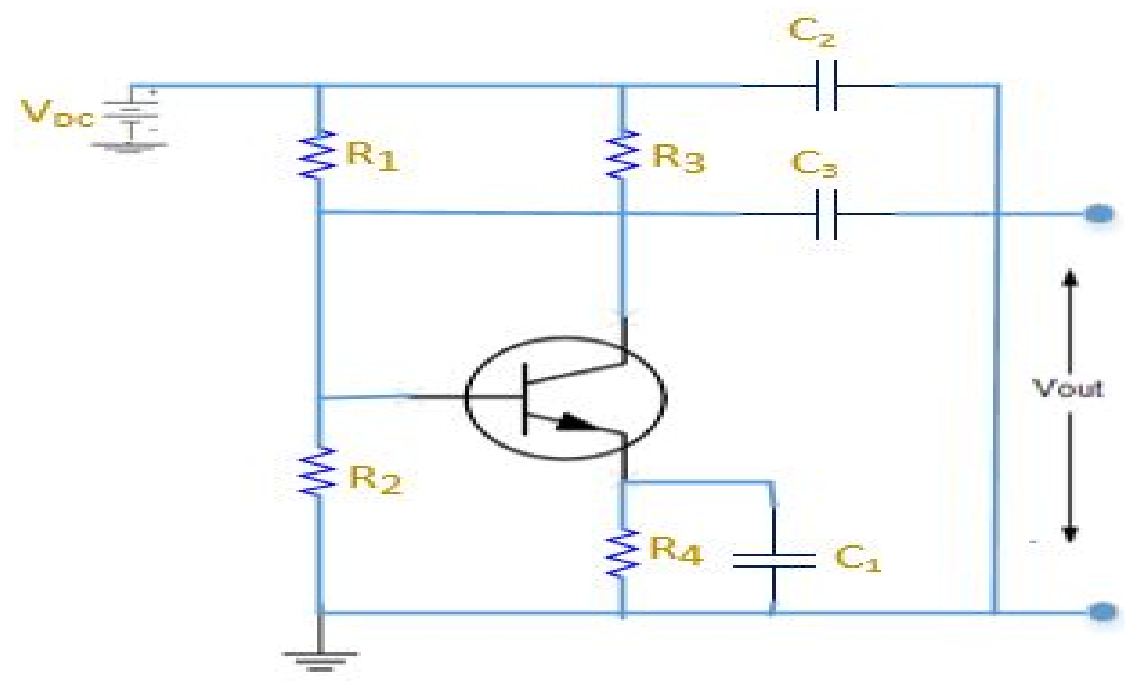

Figure (12): Clipping circuit 


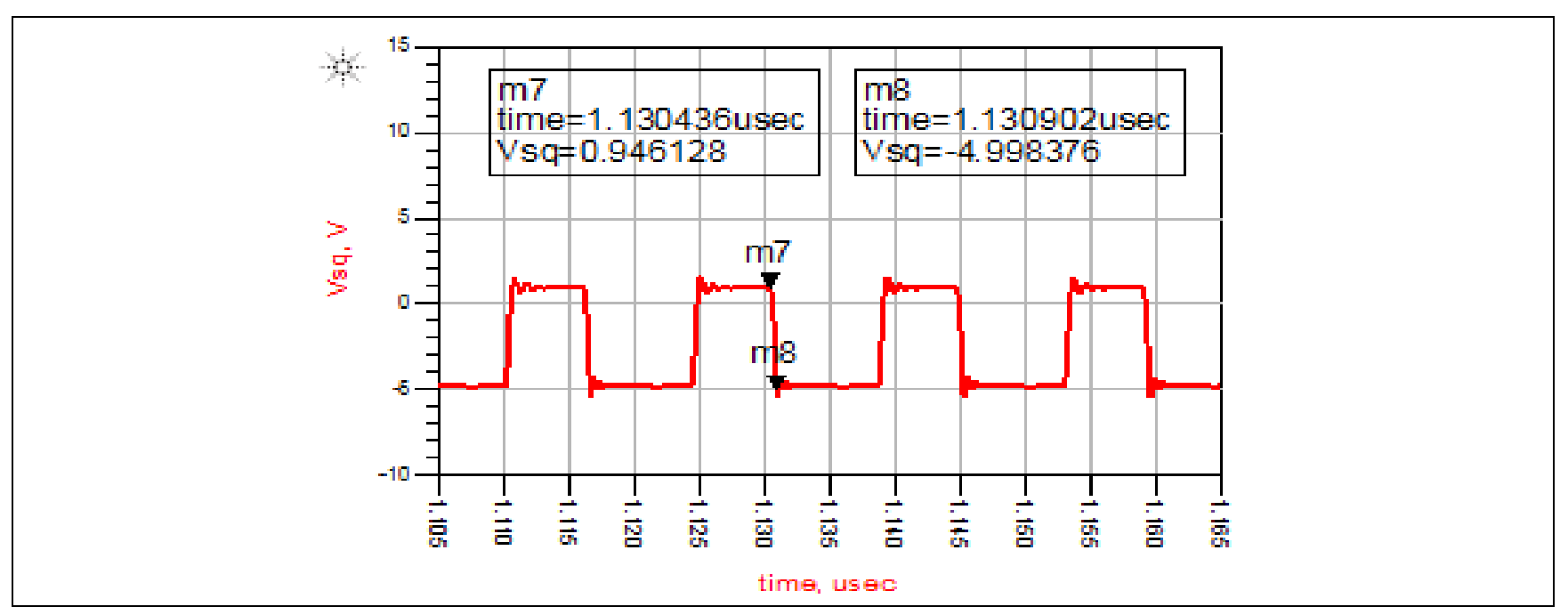

Figure (13): O/P from the clipping circuit

Fig. 14 shows the simulated monocycle pulse generated from the integrated design with 420 ps pulse width. The PSD of the output frequency is $-48.006 \mathrm{dBm} / \mathrm{MHz}$ as shown in Fig 15.

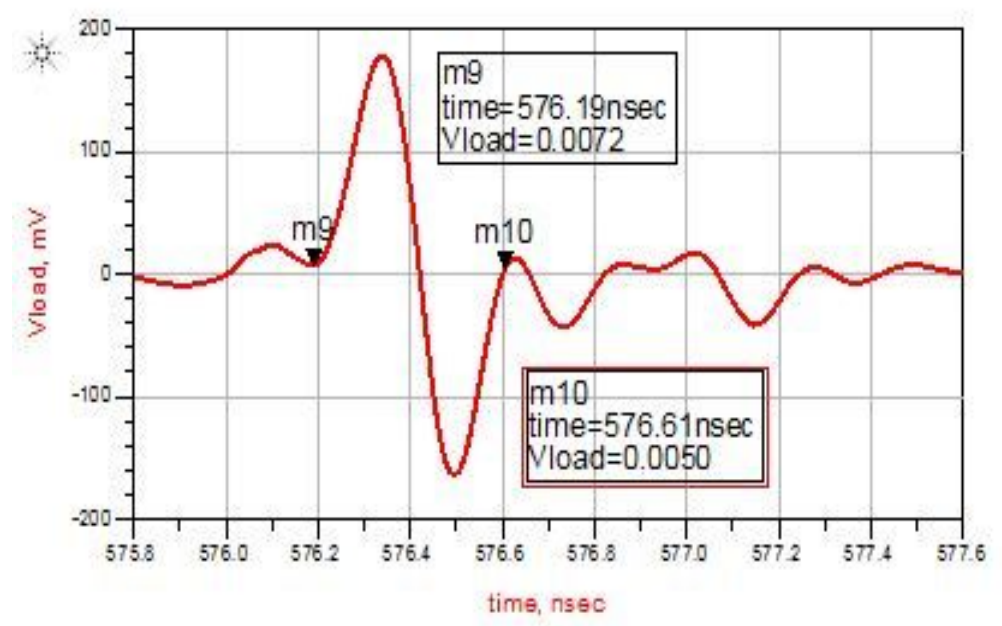

Figure (14): Monocycle pulse waveform 


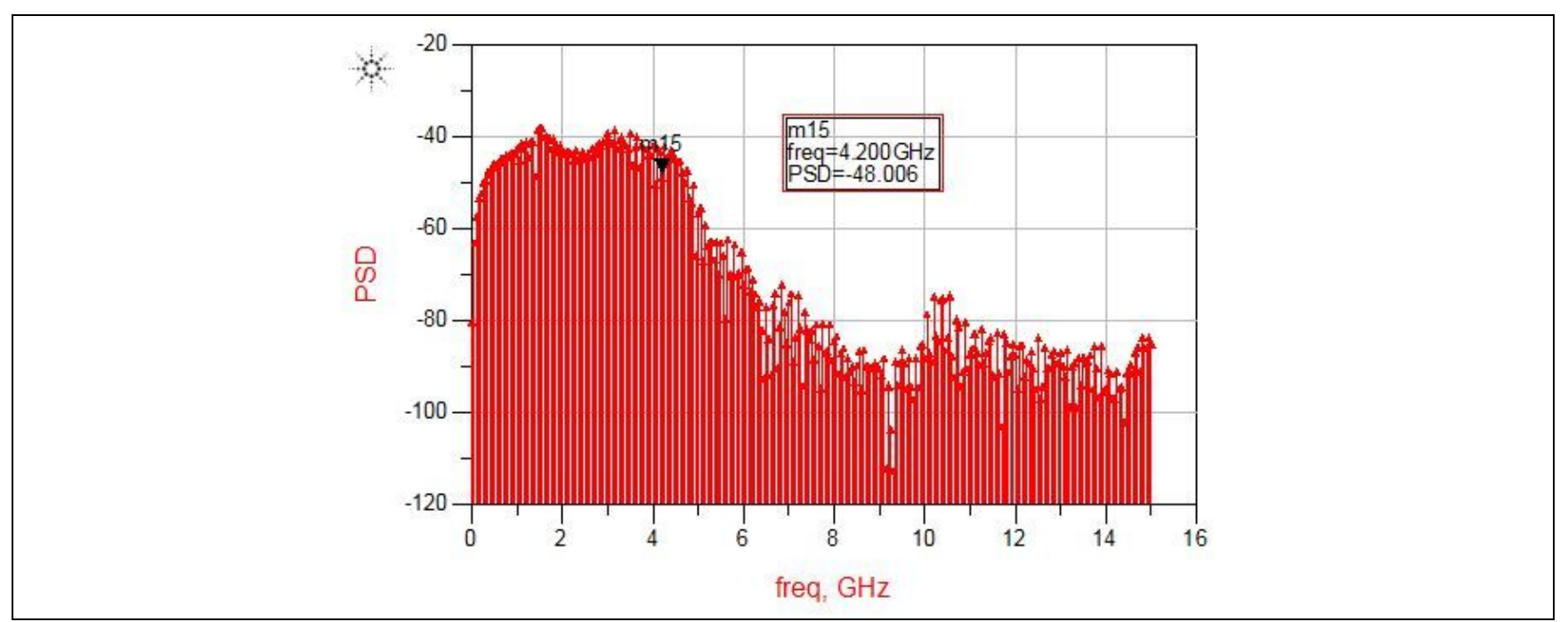

Figure (15): PSD of the monocycle pulse

\section{Conclusions:}

An UWB monocycle pulse generator using SRD has been successfully designed and fabricated. RC high-pass filter was used to apply first derivative on the Gaussian pulse. To achieve matching between Gaussian pulse generator and RC high-pass filter a shaping circuit was used. The simulated output is a monocycle pulse with 300 ps pulse width and a good symmetry in amplitude and shape. For testing purposes, a square wave signal generator was designed to trigger the monocycle pulse generator. The obtained monocycle pulse is $420 \mathrm{ps}$.

\section{References:}

[1] Faranak Nekoogar, Ultra-Wideband Communications: Fundamentals and Applications, 1st ed, September 10, 2005.

[2] Sana Ullah, Murad Ali, Asdaque Hussain, and Kyung Sup Kwak, "Application of UWB Technology," Submitted on 9 Nov 2009 (v1), last revised 2 Apr 2015 (v3), arXiv:0911.1681v3 [cs.NI].

[3] Rahayu, Y.; Rahman, T.A.; Ngah, R.; Hall, P.S., "Ultra wideband technology and its applications," in Wireless and Optical Communications Networks, 2008. WOCN '08. 5th IFIP International Conference on, vol., no., pp.1-5, 5-7 May 2008. 
[4] Dongsong Zeng; Annamalai, A.; Zaghloul, A.I., "Pulse shaping filter design in UWB system," in Ultra Wideband Systems and Technologies, 2003 IEEE Conference on , vol., no., pp.66-70, 16-19 Nov. 2003.

[5] Jeong Soo Lee; Cam Nguyen, "Novel low-cost ultra-wideband, ultra-short-pulse transmitter with MESFET impulse-shaping circuitry for reduced distortion and improved pulse repetition rate," in Microwave and Wireless Components Letters, IEEE, vol.11, no.5, pp.208-210, May 2001.

[6] Jeongwoo Han; Cam Nguyen, "A new ultra-wideband, ultra-short monocycle pulse generator with reduced ringing," in Microwave and Wireless Components Letters, IEEE, vol.12, no.6, pp.206-208, June 2002.

[7] Serres, A.; Duroc, Y.; Tan-Phu Vuong; de Farias, J.E.P.; Fontgalland, G., "A New Simple UWB Monocycle Pulse Generator," in Electronics, Circuits and Systems, 2006. ICECS '06. 13th IEEE International Conference on , vol., no., pp.12121215, 10-13 Dec. 2006.

[8] Jeongwoo Han; Cuong Huynh; Cam Nguyen, "Tunable monocycle pulse generator using switch controlled delay line and tunable RC network for UWB systems," in Antennas and Propagation Society International Symposium (APSURSI), 2010 IEEE , vol., no., pp.1-4, 11-17 July 2010.

[9] Adel S.Sedra, and Kenneth C. Smith, Microelectronic Circuits (Oxford Series in Electrical \& Computer Engineering), 6th Edition, pp. 1349-1352.

\section{Nomenclatures:}

$\mathrm{B}_{f} \ldots$ Fractional bandwidth

$\tau$... Time constant

$\mathrm{f}_{\mathrm{r}} \ldots$ Resonant frequency 